\title{
Activating killer cell lg-like receptors in health and disease
}

\author{
Martin A. Ivarsson ${ }^{1}$, Jakob Michaëlsson ${ }^{1}$ and Cyril Fauriat ${ }^{2,3,4,5,6 *}$ \\ ' Center for Infectious Medicine, Karolinska Institutet, Karolinska University Hospital Huddinge, Stockholm, Sweden \\ 2 U1068, CRCM, Immunity and Cancer, INSERM, Marseille, France \\ ${ }^{3}$ Institut Paoli-Calmettes, Marseille, France \\ ${ }^{4}$ UM 105, Aix-Marseille Université, Marseille, France \\ ${ }^{5}$ UMR 7258, CNRS, Marseille, France \\ ${ }^{6}$ U1068, CRCM, Plateforme d'Immunomonitoring en Cancérologie, INSERM, Marseille, France
}

\section{Edited by:}

Simona Sivori, University of Genoa,

Italy

\section{Reviewed by:}

Michela Falco, Istituto Giannina Gaslini, Italy

Christelle Retière, Etablissement

Français du Sang, France

*Correspondence:

Cyril Fauriat, Institut Paoli-Calmettes, Aix-Marseille Université, CRCM,

Immunity and Cancer, INSERM,

U1068, UM 105, Marseille F-13009,

France; CNRS, UMR 7258, Marseille

F-13009, France

e-mail:cyril.fauriat@inserm.fr

Expression of non-rearranged HLA class I-binding receptors characterizes human and mouse NK cells. The postulation of the missing-self hypothesis some 30 years ago triggered the subsequent search and discovery of inhibitory MHC-receptors, both in humans and mice. These receptors have two functions: (i) to control the threshold for NK cell activation, a process termed "licensing" or "education," and (ii) to inhibit NK cell activation during interactions with healthy HLA class l-expressing cells. The discovery of activating forms of KIRs (aKIR) challenged the concept of NK cell tolerance in steady state, as well as during immune challenge: what is the biological role of the activating $\mathrm{KIR}$, in particular when NK cells express aKIRs in the absence of inhibitory receptors? Recently it was shown that aKIRs also participate in the education of NK cells. However, instead of lowering the threshold of activation like iKIRs, the expression of aKIRs has the opposite effect, i.e., rendering NK cells hyporesponsive. These findings may have consequences during NK cell response to viral infection, in cancer development, and in the initial stages of pregnancy. Here we review the current knowledge of activating KIRs, including the biological concept of aKIR-dependent NK cell education, and their impact in health and disease.

\section{Keywords: NK cells, KIR, HLA, education, infection diseases, cancer, pregnancy, autoimmune diseases}

\section{INTRODUCTION}

The history of non-rearranged MHC class I-binding receptors has been a passionate story for three decades. Shortly after the discovery of NK cells in the mid-1970s $(1,2)$ MHC class I was suspected to be important for regulating NK cell responses (3-5). The subsequent postulation of the "missing-self" hypothesis $(1,2,6)$, predicted that NK cells would express inhibitory MHC class I-binding receptors. In 1992, the discovery of inhibitory MHC class I-binding Ly49 receptors in mice transformed the "missing-self" hypothesis into a now well-admitted dogma $(3-5,7)$. Shortly after, human inhibitory receptors binding to HLA-C (KIR2DL1/P58.1/EB6 and KIR2DL3/P58.2/GL183) (8-10) and HLA-B (NKB1/KIR3DL1) (11), were identified as functional analogs to the murine inhibitory Ly49 receptors. Moretta and Colleagues later discovered that the monoclonal antibody clone EB6 also could recognize a second receptor p50 (KIR2DS1), and that this receptor transmitted activating signals upon recognition of HLA-Cw4 targets (12). The genes coding for these receptors were later cloned and were confirmed to be specific for HLA-B and -C molecules (13). Since the initial discovery of the first inhibitory and activating KIRs in humans (referred to iKIRs and aKIRs respectively in the rest of the manuscript), a number of additional KIRs have been identified. The KIR family now includes seven iKIR and six aKIR, in addition to KIR2DL4, which has both inhibitory and activating function. Adding to the complexity, each KIR gene is highly polymorphic, and the product of different alleles can interact more or less strongly with different HLA class I alleles (Table 1).
All KIR genes are encoded in the leukocyte receptor complex (LRC) in the chromosome 19 (19q14.3). Thus, the LRC includes the 15 genes of the KIR family. Importantly, for some KIRs, there is extreme allele variability, whereas some KIR genes are highly conserved and exist only as few alleles. The variability of KIR alleles can be nicely visualized ${ }^{1}$. Interestingly, compared to iKIR, aKIR display rather limited allele variability compared to iKIR. For instance there are more than 75 alleles coding for KIR3DL1 and only 17 coding for KIR3DS1 (32), and KIR2DS1 gene encompassed only 16 alleles in contrast to KIR2DL1 gene, which include more than 40 alleles ${ }^{2}$.

In addition to allele polymorphism, there is strong haplotype variability due to the number of KIR genes present in the LRC. Two types of KIR haplotypes, A and B, have been defined pending on gene content. Group B haplotypes are defined by the presence of at least one of the following KIR genes: KIR2DL5, KIR2DS1, KIR2DS2, KIR2DS3, KIR2DS5, and KIR3DS1. Conversely, group A haplotypes are characterized by the absence of all these genes. All information about KIR alleles and haplotypes has been collected in the KIR web-based database (see text footnote 1). In addition, another repository has been created for association between KIR and diseases $^{3}(33)$.

Currently, the aKIR include KIR2DS1, KIR2DS2, KIR2DS3, KIR2DS4, KIR2DS5, and KIR3DS1 (Table 1). The specificity of

\footnotetext{
${ }^{1}$ http://www.allelefrequencies.net/kir6008a.asp

${ }^{2}$ http://www.ebi.ac.uk/ipd/kir/

${ }^{3}$ http://www.allelefrequencies.net/diseases/default.asp
} 
Table 1 | Activating and inhibitory KIRs and ligands.

\begin{tabular}{|c|c|c|c|}
\hline Activating KIR & Ligand & Detectable by FACS & Reference \\
\hline 2DS1 & HLA-C2 (weaker than 2DL1) & Yes & $(14,15)$ \\
\hline 2DS2 & HLA-C1 (weak), HLA-A*11:01 & Yes & $(16-18)$ \\
\hline 2DS3 & Unknown & ? (Retained intracellularly?) & (19) \\
\hline 2DS4 & HLA-C*05:01, A*11:02, C*16:01 & Yes & $(20)$ \\
\hline 2DS5 & Unknown & Yes & $(21-23)$ \\
\hline 3DS1 & Unknown & Yes & $(24)$ \\
\hline Inhibitory KIR & Ligand & Detectable by FACS & \\
\hline 2DL1 & HLA-C2 (N77/K80) & Yes & (8) \\
\hline 2DL2 & HLA-C1 (S77/N80), HLA-C2, HLA-B*46:01 and HLA-B*73:01 (C1 epitope) & Yes & $(9,25)$ \\
\hline 2DL3 & HLA-C1 (S77/N80), HLA-C2, HLA-B*46:01 and HLA-B*73:01 (C1 epitope) & Yes & $(9,25)$ \\
\hline 2DL4 & HLA-G (intracellular interaction?) & Yes (not shown in vivo) & $(26)$ \\
\hline 2DL5A/B & Unknown & Yes & $(27)$ \\
\hline 3DL1 & HLA-A (with Bw4 motif), HLA-Bw4 & Yes & $(11,28)$ \\
\hline 3DL2 & HLA-A3/A11 & Yes & $(29,30)$ \\
\hline 3DL3 & Unknown & ? (Methylated promoter) & (31) \\
\hline
\end{tabular}

each receptor as well as their cell surface expression has been elusive for many years, despite the fact that the extracellular domains of these molecules are extremely similar to their inhibitory counterparts, in terms of sequence and 3D structure. Studies implicating aKIRs in diseases are therefore almost exclusively based on genetic studies. More recently, new staining protocols and monoclonal antibodies have allowed identification of cells expressing KIR2DS1, KIR2DS2, KIR3DS1, and KIR2DS5 (15, 16, 22-24, 3438). The possibility to identify the specific expression of distinct aKIRs has made it possible to determine how these receptors regulate NK cell function at the single level, and in concert with other activating and inhibitory HLA class I-binding receptors.

KIR2DL4 remains a particular KIR because of its structure, expression, and functions (39). Hence, this receptor is expressed by all NK cells, in contrast to other KIRs, which are clonally expressed. KIR2DL4 has also a lower diversity but its allele diversity influences the surface expression and function in NK cells $(40,41)$. Hence, the 9A allele of KIR2DL4 is not stable at the plasma membrane and is rapidly recycled. Consequently, intracellular KIR2DL4, engagement with its ligand HLA-G in endocytosis vesicles resulted in production of cytokines such as IFN- $\gamma$ but also other cytokines such as IL-1 $\beta$, TNF- $\alpha$, and IL-8, etc., [nicely reviewed by Rajagopalan and Long (39)].

\section{THE ELUSIVE NATURE OF ACTIVATING KIR LIGANDS}

Despite the high degree of sequence homology between activating and inhibitory KIRs, the specificities of most aKIRs remain elusive. In particular, this is true for KIR2DS1, KIR2DS2, and KIR3DS1. KIR2DS1, the first described, and most well-studied activating KIR, binds to HLA-C molecules within the $\mathrm{C} 2$ group (with the N77/K80 motif) (12). KIR2DS2 has been shown to recognize group 1 HLA-C and induce a KIR-HLA-dependent NK cell activation $(42,43)$. However, many other studies have failed to identify KIR2DS2-HLA-C1 interaction [nicely reviewed by Moesta and Parham (44)]. Unexpectedly, a recent publication revealed that KIR2DS2 recognizes HLA-A ${ }^{\star} 11$ (18). With respect to KIR3DS1, while there are indications that KIR3DS1 interacts with HLA-Bw4 $4^{80 \mathrm{I}}$, there have been no studies demonstrating a direct interaction between these two molecules (24). In contrast to the above-mentioned aKIRs, KIR2DS3 and KIR2DS5 have no inhibitory counterparts. Although detection at the cell surface of NK cells has been recently proven possible $(19,21)$, the identification of their respective ligand(s) has not been successful. KIR2DS4 however has been shown to interact with HLA- ${ }^{\star} 11: 02$, HLA-C ${ }^{\star} 05: 01$, and HLA-C*16:01 (20).

It is possible that the aKIRs have HLA class I as their major ligands. However, it also remains possible that these receptors could recognize altered HLA class I complexes, e.g. specific HLA/peptide complexes, or complexes of HLA class I together with viral proteins, or non-HLA class I ligands altogether (45). For example, KIR2DS4 has been suggested to bind to an unidentified protein expressed on melanoma-derived tumor cells, independently of HLA class I (46). In mice, the activating Ly49H receptor directly recognizes the CMV-encoded protein $\operatorname{m} 157$ (47, 48), and thereby allows NK cells to control CMV infection. Similarly, mouse CMV m04 together with the MHC class I molecule $\mathrm{H}-2 \mathrm{D}^{\mathrm{k}}$ allows recognition of CMV-infected cells by NK cells expressing the activating receptor Ly49P (49). It thus remains possible that the aKIRs could directly, or indirectly, recognize virally encoded ligands, although such ligands have yet to be identified in humans.

Several iKIRs display a certain degree of peptide selectivity, where the nature of the HLA class I presented peptide affects the binding to KIRs $(43,45,50,51)$. Similarly, KIR2DS1 displays a certain degree of peptide selectivity in its binding to HLA-Cw4 (43), indicating that also aKIRs can be modulated by the nature of the presented peptide. Noteworthy the recent crystal structure of KIR2DS2-HLA-A ${ }^{\star} 11: 01$ was obtained in complex with a vaccinia viral peptide (18). Moreover, the authors showed that peptide sequence affects the binding of KIR-HLA. Additionally, HLA 
allele themselves can display a different affinity for their attributed KIR (42).

In addition to alterations in peptide content during, e.g., viral infections, the overall cell surface pattern of MHC class I can be altered. Interestingly, it was recently shown in mouse, that peptidespecific clusters of HLA class I/peptide complexes are formed on the cell surface of infected cells, depending on the nature of the peptide presented (52). These peptide-specific clusters create a high level of expression of a given MHC class I/peptide complex on the cell surface of infected cells, which allow a more efficient recognition by cognate $\mathrm{T}$ cell receptors (52). It is tempting to speculate that in humans, the clustering of specific HLA class I/peptide complexes during infections could be one way for aKIRs to recognize virus-infected cells. Noteworthy, most studies on aKIRs have evidenced a weaker affinity for their ligands compared to their inhibitory counterparts $(43,44)$.

\section{EDUCATION OF NK CELLS BY ACTIVATING KIR}

Activating KIRs convey their signals through the adaptor KARAP/DAP12 $(53,54)$. Upon ligation with antibodies or ligands, Src kinase-dependent phosphorylation of DAP12 allows the recruitment of SYK and the triggering of LAT-dependent signaling pathways (PI3K-AKT, NKFAT, and MAPK) (55).

As mentioned above, the ligands for most aKIRs remain elusive and KIR2DS1 is the activating receptor with the best described ligand (HLA-C2) $(43,56,57)$. Several studies have shown that KIR2DS1 ${ }^{+}$NK cells efficiently kill HLA-Cw4 ${ }^{+}$target cells (12, $14,15,43,45,58)$. However, it has been hard to reconcile the fact that NK cells can express activating receptors whose ligand is expressed on healthy cells, as this interaction potentially could lead to autoreactivity. To cope with potential autoreactivity, it is likely that NK cells, much like T cells, have evolved a system for tolerance also for aKIRs. One possibility is negative selection of NK cells expressing self-HLA class I-binding aKIRs in the absence of self-specific inhibitory KIRs. However, recent data refuted this hypothesis, as NK cells expressing KIR2DS1 in the absence of self-specific inhibitory KIRs or CD94/NKG2A can be detected in HLA-C2 ${ }^{+}$donors $(15,36,37,59)$.

An alternative hypothesis is that NK cells expressing aKIRs are not deleted, but rather rendered hyporesponsive if the ligand is present in the host. Indeed, in HLA-C2 homozygous donors NK cells expressing KIR2DS1 in the absence of self-HLA class I-specific KIRs (KIR2DS1sp) were hyporesponsive to target cell stimulation, whereas no such hyporesponsiveness was observed in HLA-C1 homozygous donors (15), indicating that the responsiveness of KIR2DS1 ${ }^{+}$NK cells is tuned down in HLA-C2 homozygous donors $(15,59)$. Similarly, IL-2 activated KIR2DS1sp NK cell lines and clones from HLA-C2 homozygous donors have been reported to have lower responses to HLA-C2 ${ }^{+}$target cells $(14,59)$.

This newly identified NK cell education complements education via inhibitory HLA class I-binding receptors (iKIR and NKG2A/CD94), since it renders otherwise potentially autoreactive NK cells self-tolerant. The system of iKIR- and aKIR-mediated education can in some ways be compared to the positive and negative selection of T cells; where $\mathrm{T}$ cells are positively selected based on having TCRs that recognize self-MHC class I, and negatively selected if they express a TCR that recognize MHC class
I in complex with self-peptides. In comparison with the T cells, education by iKIR would be the analog of positive selection, and education via aKIRs would be the analog of negative selection, with the major difference that $\mathrm{T}$ cell selection results in deletion or survival, whereas for NK cells it sets the threshold for activation, without affecting survival $(60,61)$.

Interestingly, although the mechanisms have not yet been identified, education by aKIRs shares features with the hyporesponsiveness induced by chronic stimulation of other activating receptors expressed by NK cells. For example, chronic exposure to NKG2D ligands in mice renders NK cells hyporesponsive to target cells (62). Similarly, when the ligand (m157) for the activating $\mathrm{Ly} 49 \mathrm{H}$ is constitutively expressed, mouse $\mathrm{Ly}_{49 \mathrm{H}^{+}} \mathrm{NK}$ cells become hyporesponsive (63). Finally, NK cells in NKp46-deficient mice are more responsive, suggesting that NKp46 hampers the reactivity of NK cells via an unidentified constitutively expressed ligand (64). Thus, the mechanism ensuring tolerance by self-HLA class I-binding aKIRs might be similar to that of these activating receptors. Other examples of activating receptor-dependent NK cell tolerance exist but the molecular mechanisms are likely to be different. For instance, mouse 2B4 may be associated with different adaptors that transmit inhibitory or activating signals (65). Interestingly, the tuning of NK cells expressing self-HLA class I-binding aKIRs seems to be restricted to target cell recognition, as it does not affect the response to cytokine stimulation (15). Furthermore, based on murine studies, education of NK cells appears to be a dynamic and reversible process, both via activating and inhibitory receptors $(66,67)$. This suggests that NK cells expressing self-HLA class I-binding receptors may be silenced under normal conditions, but once an appropriate signal is given to the cells, they may become an important component of an efficient immune response. For example, sudden changes, in contrast to chronic long-term changes, might provide such a signal, a concept that was recently proposed in the "discontinuity theory for immunity" (68). Compiling all relevant literature, the authors noted that innate immune cells, but also to some extent adaptive immune cells, are tolerant to "self," irrespective of how aberrant the "self" is, until an "unexpected" event disrupts the equilibrium that is maintaining tolerance, thus awakening the immune effectors.

Overall, the iKIR- and aKIR-dependent education of NK cells by HLA class I molecules, together with the polymorphic nature of KIRs and HLA class I, and the variegated expression of KIRs on NK cells, results in a highly complex system of NK cell regulation. The effects of this complex system are only just starting to be investigated in different diseases, and most available data thus comes from genetic studies.

\section{ACTIVATING KIRS IN VIRAL INFECTIONS}

Over the past 10 years, a number of studies have aimed at elucidating a potential role of aKIRs in virus infections, including $\mathrm{HIV}, \mathrm{HCV}$, and CMV. There is now compelling evidence, although mostly at the genetic level, that aKIRs can affect the outcome of different infections. The large degree of linkage disequilibrium between different KIR genes, e.g., KIR2DL2 and KIR2DS2, and KIR2DS1 and KIR3DS1, however makes it hard to pinpoint a specific KIR/HLA class I interaction at the genetic level, as responsible for protection or susceptibility to a given infection. In addition, 
the lack of well-characterized ligands for several of the aKIRs, e.g., KIR3DS1, KIR2DS2, and KIR2DS3, as well as the lack of specific monoclonal antibodies to these aKIRs have severely hampered investigations of the functional role of these receptors in viral infections. The identification of ligands and the development of new antibodies specific for aKIRs are thus required to move the field forward toward understanding how these receptors regulate NK cell function in different infectious disease settings.

KIR3DS1 is the by far most well-studied aKIR in the setting of infectious diseases [also nicely reviewed by Körner and Altfeld (69)]. The first evidence for a role of KIR3DS1 in viral infections came from genetic studies in HIV-infected individuals where individuals homozygous for KIR3DS1 progressed slower to AIDS, but only if they also carried $H L A-B w 4$ with an isoleucine at position 80 (HLA-Bw $4^{80 I}$ ) (70). Interestingly, donors homozygous for KIR3DS1, but lacking HLA-Bw $4^{80 I}$ progressed faster to AIDS (70). Furthermore, $H L A-B w 4^{80 I+}$ individuals with increasing number of copies of KIR3DS1 due to copy number variation have been shown to have lower viral load set points (71). The frequency of KIR3DS1 positive individuals has also been shown to be higher in HIV-exposed seronegative individuals, compared to HIV seropositive and HIV negative individuals, indicating that KIR3DS1 might also be providing protection against infection (72). In addition,

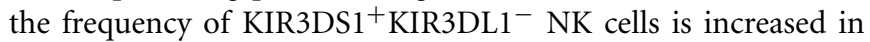
$H L A-B w 4^{80 I+}$ individuals, both at acute and chronic stages of HIV infection compared to healthy controls (73). The protective effect of KIR3DS1 in HIV infection was further strengthened by evidence that individuals carrying both KIR3DS1 and HLA$B w 4^{80 I}$ suppress HIV replication in vitro to a greater extent than NK cells from individuals carrying KIR3DS1 in the absence of $H L A-B w 4^{80 I}$, or vice versa, carrying $H L A-B w 4^{80 I}$ in the absence of KIR3DS1 (56). In addition, purified KIR3DS1 ${ }^{+}{\mathrm{KIR} 3 \mathrm{DL} 1^{-}}^{-} \mathrm{NK}$ cells from $H L A-B w 4^{80 I+}$ individuals suppressed HIV replication in vitro to a much greater extent than $\mathrm{KIR}_{3} \mathrm{DS} 1^{-} \mathrm{KIR}^{-} \mathrm{DL} 1^{+}$and KIR3DS1 ${ }^{-} \mathrm{KIR} 3 D L 1^{-}$NK cells (56), indicating that the effect of KIR3DS1 is likely to be mediated by KIR3DS $1^{+} \mathrm{NK}$ cells, and not by KIR3DS1 ${ }^{+} \mathrm{T}$ cells. The same group subsequently demonstrated that the effect of KIR3DS1 on viral inhibition in vitro was highest in individuals carrying KIR3DS1, HLA-Bw $4^{80 I}$, and at least one copy of KIR3DL1 (71). Surprisingly, NK cells from $H L A-B w 4^{80 I+}$ individuals homozygous for KIR3DS1, but lacking KIR3DL1, did not suppress HIV replication in vitro to a greater extent than NK cells from $H L A-B w 6$ homozygous individuals, indicating that the effect of KIR3DS1 is dependent on both KIR3DL1 and HLA-Bw4 ${ }^{80 I}$. Interestingly, the expression of KIR3DS1, both at the mRNA and protein level, was elevated in individuals carrying KIR3DS1 in the presence of two copies of KIR3DL1, compared to individuals carrying KIR3DS1 in the presence of one copy of KIR3DL1. However, that study did not compare the expression levels of KIR3DS1 to individuals carrying two copies of KIR3DS1 in the absence of KIR3DL1, making it hard to determine whether increased expression levels of KIR3DS1 alone could explain the increase in viral inhibition, as suggested by the authors. An alternative explanation for the increased suppression of HIV replication by KIR3DS1/KIR3DL1 heterozygous individuals, compared to KIR3DS1 homozygous individuals could be an increased efficiency of NK cells co-expressing KIR3DL1 and
KIR3DS1, potentially allowing NK cells to sense a down regulation of HLA-Bw4 via KIR3DL1, and at the same time activate them via KIR3DS1-mediated recognition of HLA-Bw4 with, e.g., an altered peptide repertoire. However, sorted KIR3DL1 ${ }^{+} \mathrm{NK}$ cells from KIR3DS1/KIR3DL1 heterozygous individuals, that could contain NK cells co-expressing KIR3DL1 and KIR3DS1, did not efficiently suppress HIV replication in vitro, compared to NK cells expressing only KIR3DS1 (56). This indicates that co-expression of KIR3DL1 and KIR3DS1 is not required for KIR3DS1-mediated NK cell suppression of HIV replication in vitro. It is also noteworthy that very little is known about co-expression of other activating and inhibitory KIRs and other HLA class I-binding receptors (e.g., NKG2A, NKG2C, and LIR-1) on KIR3DS1 ${ }^{+}$NK cells, both in healthy and in HIV-infected individuals.

The complexity of the effects of KIR3DS1 in HIV infection is further highlighted by reports that KIR3DS1 is associated with an increased progression to AIDS, but when restricted to $H L A-B w 4^{+}$ individuals, KIR3DS1 presence is associated with a slower progression (74). Other reports have suggested an HLA-Bw4-independent effect of KIR3DS1 in HIV-infected individuals, at least when measuring CD8 T cell activation, which is strongly associated with HIV disease progression (75). Taken together, the data collected to date indicate a role for KIR3DS1 in HIV disease and progression to AIDS, but it is also clear that more efforts are needed to explain the complex nature of KIR3DS1-mediated protection in $\mathrm{HIV}$-infected individuals, including the identification of a ligand for this receptor.

In contrast to the role of KIR3DS1 in HIV infection, much less is known about the role of other aKIRs in viral infections. Similar to studies of aKIR and HIV, most of the studies have been performed at the genetic level, and commonly in smaller cohorts of patients. Nevertheless, a number of studies have implicated a role for aKIRs in other viral infections. For example, the frequency of KIR2DS3 positive individuals is higher in patients with chronic HCV infection, compared to individuals that have resolved HCV infection (76). Interestingly, the detrimental effect of KIR2DS3 was only observed in $H L A-C 2^{+}$individuals, which is not believed to be a ligand for KIR2DS3 (76). An increase in KIR2DS3, as well as KIR2DS2 positive individuals has also been reported in a Brazilian cohort of chronically HCV-infected patients (77). Whether the association between KIR2DS3 and chronic HCV infection reflects a direct interaction between HCV-infected cells and KIR2DS3 ${ }^{+}$NK cell remains unknown since there is neither a known ligand, nor specific antibodies for KIR2DS3. A likely explanation proposed by Dring et al. is that KIR2DS3 is instead a marker of a particular haplotype that is associated with the disease progression (76).

In addition to a role for KIR3DS1 in HIV infection, KIR2DS2 has been associated with faster progression to AIDS (74). A similar association was found in a West African cohort of HIV-infected women, where haplotype B KIRs, including KIR2DS2, KIR2DS3, and KIR3DS1, was associated with lower CD4 T cell counts (78). Interestingly, homozygosity for $H L A-C 1$, a putative ligand for KIR2DS2 and KIR2DS3, decreased the association between lower CD4 T cell counts and the aKIRs. However, there is no evidence for a direct functional role of KIR2DS2 or KIR2DS3 in recognition of HIV-infected cells. Because HLA-C is not downregulated by HIV-infected cells (79), it is tempting to speculate that NK 
cells expressing HLA-C-binding aKIRs in the absence of HLA-Cbinding iKIRs, could mediate recognition of HIV-infected cells. For example, KIR2DS1 ${ }^{+} \mathrm{KIR}_{2} \mathrm{DL} 1^{-} \mathrm{NK}$ cells, which are activated by interaction with HLA-C2 ${ }^{+}$target cells, could potentially recognize HIV-infected cells in HLA-C2 ${ }^{+}$individuals. The lack of effects of KIR2DS1/HLA-C2 in HIV infection could potentially be explained by the education of NK cells via aKIRs, where KIR2DS1 ${ }^{+}$ NK cells in HLA-C2 ${ }^{+}$individuals are hyporesponsive.

In addition to the associations between activating KIRs and viral infections described above, KIR2DS1 and KIR2DS3 have also been associated with fatal outcome in Ebola infection. The frequencies carriers of both these genes were increased in patient cohorts with fatal outcome of Ebola infections, compared to survivors, contacts, and healthy controls (80). However, as the study investigated a rather small cohort, no further dissection of interactions between these aKIRs and HLA-C was possible. Expansions of KIR2DS2 ${ }^{+}, \mathrm{KIR}_{2} \mathrm{DS} 4^{+}$, and KIR3DS1 ${ }^{+}$NK cells have also been reported to occur in a subset of CMV seropositive individuals (17). However, direct evidence for a role of these aKIRs in the recognition of CMV-infected cells is still lacking.

In summary, although there are a fairly large number of studies associating KIR gene content with outcome of viral infections, more direct functional evidence for a role of aKIRs in recognition of virus-infected cells is lacking. The identification of novel, possibly virus-encoded or virus-induced, ligands for activating KIRs, as well as specific monoclonal antibodies, are required to understand the role of these receptors in viral infections.

\section{ACTIVATING KIR IN CANCER}

A number of studies have investigated the associations between inhibitory and activating KIR and cancer prognosis. However, similar to the role of aKIRs in infections, the evidence for a role for aKIRs in cancer has largely been at the genetic level due to the limitations in available specific monoclonal antibodies. The education of NK cells via aKIR/HLA class I may play a significant role in controlling NK cell responses to transformed cells, as NK cells expressing aKIRs are hyporesponsive in the presence of self-HLA class I ligands. NK cells expressing aKIR in individuals where the ligand is present will be hyporesponsive, and thus may not be able to mount an efficient response against tumor cells in the same individual. Conversely, NK cells expressing aKIRs in individuals where the ligand is not expressed will not be able to recognize the tumor cells due to the lack of the ligand. However, it remains possible that there are hitherto unknown ligands for aKIRs expressed on tumor cells (46), potentially providing a role for aKIRs in various cancers. A recent study evidenced that CCR7 expressed by dendritic cells or lymphoblasts could be transferred to NK cells in a KIR2DS1/HLA-C2-dependent manner. Consequently, $\mathrm{CCR7}^{+}$NK cells would migrate to secondary lymphoid organs and further improve anti-leukemic effect of NK cells, notably after hematopoietic stem cell transplantation (haplo-HSCT) (81).

The role of activating KIRs in cancer seems to vary with type of cancer, therapy and clinical measurement, and evidence for both protective and detrimental effects exist. For example, KIR2DS1 gene frequency was found to be higher in breast cancer patients compared to healthy controls, and in contrast to KIR2DL1 was suggested to promote cancer progression (82). Similarly, individuals with chronic myeloid leukemia carrying the KIR2DS1 gene had a lower response to antibody-treatment with Imatinib (Glivec) (83). In addition, multiple myeloma patients carrying KIR3DS1 had shorter progression-free survival (PFS), compared to those that lacked KIR3DS1, after autologous stem cell transplantation (84). However, among the KIR3DS1 positive individuals in that study, patients carrying $H L A-B w 4$ had longer PFS compared to those lacking $H L A-B w 4$. In contrast, Karabon et al. recently reported that KIR3DS1 positive individuals with B cell lymphocytic leukemia (B-CLL) had a trend toward longer progression-free survival compared to KIR3DS1 negative individuals. Interestingly however, in the same study co-carriage of KIR3DS1 among HLA-Bw $4^{+}$ patients, as well as co-carriage of KIR2DS1 in $H L A-C 2^{+}$patients were associated with longer PFS (85).

Allogeneic stem cell transplantation to treat leukemia is one setting where NK cells expressing aKIRs may play a significant role, in particular when using KIR ligand mismatched donor/recipient pairs. In this setting, aKIR ${ }^{+}$NK cells from donors lacking expression of ligands for the aKIRs could potentially recognize the recipients' leukemia blasts, e.g., when transplanting a HLA-C2 positive recipient with cells from a KIR2DS1 positive but HLA$C 2$ negative donor. Indeed, it was recently shown in acute myeloid leukemia that patients receiving allografts from KIR2DS1 positive donors had a lower probability of relapse, but only if the donor was not homozygous for HLA-C2 (86). Interestingly, there was no difference in relapse between $H L A-C 2$ homozygous patients receiving a KIR2DS1 positive allograft, compared to those receiving a KIR2DS1 negative allograft. In contrast, there was a lower probability of relapse in $H L A-C 1 / C 1$ and $H L A-C 1 / C 2$ patients receiving a KIR2DS1 positive allograft, compared to those receiving a KIR2DS1 negative allograft (86). Based on their data, the authors concluded that it would be beneficial to select KIR2DS1 positive $H L A-C 1$ positive donors for transplantation into HLAC1 positive donors, and suggested that tolerance-induction via KIR2DS1/HLA-C2 could potentially explain the lack of effects when the allograft was derived from KIR2DS1 positive HLAC2/C2 donors. The data are also compatible with KIR2DS1 ${ }^{+}$ donor NK cell education via interactions with HLA-C2 in the recipient, since there was no effect of donor KIR2DS1 in HLAC2/C2 recipients. However, it is hard to reconcile how KIR2DS1 ${ }^{+}$ NK cells could mediate an anti-leukemic effect when transplanted into $H L A-C 1$ positive donors, as there would be no ligand for KIR2DS1 expressed by the recipient's leukemia blasts. One possible explanation, which is still compatible with aKIR-mediated education, is that an unknown non-HLA class I ligand is expressed by leukemic blasts, and as such provides a mechanism by which KIR2DS1 ${ }^{+}$NK cells could recognize the leukemia blasts, but only if the donor-derived KIR2DS1 ${ }^{+} \mathrm{NK}$ cells are not rendered tolerant by the donors or recipient's HLA-C2. However, no such ligands been identified to date. In addition to the study by Venstrom et al. (86), several other studies have indirectly indicated an effect of activating KIR in hematopoietic cell transplantation. In a study with over 1000 AML patients, receiving grafts from KIR haplotype B donors, in particular KIR cen-B homozygous donors, was associated with a higher relapse-free survival, compared to those receiving grafts from KIR haplotype A/A donors, which lack most aKIRs $(87,88)$. Interestingly, no effect of donor KIR haplotype B 
was observed on outcome of ALL in the same study. In contrast to these studies, McQueen et al. reported that KIR haplotype A/A patients with AML/MDS receiving KIR haplotype B grafts (containing aKIR) had a lower survival together with a higher relapse and GvHD rates, compared to KIR haplotype A/A donors receiving a KIR haplotype A/A graft (89). It should however be noted that the number of patients with KIR haplotype A/A receiving a KIR haplotype B graft in that study was low (8-11 patients), making it hard to draw firm conclusions.

More direct evidence for a role of aKIRs in tumor recognition was provided by Pende et al. in patients receiving haploidentical haplo-HSCT from KIR ligand-mismatched donors. Donor-derived KIR2DS1 ${ }^{+}$NK cells were shown to efficiently kill HLA-C2 ${ }^{+}$leukemia blasts, indicating that aKIRs could mediate important anti-leukemic effects in the setting of bone marrow transplantation (90).

Overall, although a number of studies have analyzed the cocarriage of KIR genes and their ligands, general conclusions regarding the role of aKIR in cancer and cancer therapy cannot be drawn, as it is likely that the role of aKIRs will vary depending on the type of cancer and treatment. Given the linkage disequilibrium of activating KIRs, large cohorts of well-defined patients are needed to pinpoint the role of specific aKIRs, as well as the presence of their ligands. In addition, functional data investigating the role of aKIRs, and education via these receptors, is largely lacking. More studies monitoring aKIR-dependent NK cell functions are thus warranted in order to decipher the impact of aKIR-mediated education on cancer incidence and progression.

\section{ACTIVATING KIR IN HUMAN REPRODUCTION}

More and more studies reveal the presence of NK cells in tissue and in particular uterine NK (uNK) cells have been found highly interesting with regard to their regulation by aKIRs. uNK cells represent the dominant leukocyte subset in the decidua (uterine endometrium in pregnancy) during the initial period of pregnancy (91). The uNK cells have been linked to the particular physiological changes that the implantation of the embryo to the uterine wall entails [elegantly reviewed by Mofett and Loke (92)]. Briefly, during the initial stages of pregnancy, fetal extravillous trophoblasts (EVT) implant the embryo by invading into the maternal uterine wall and remodel uterine spiral arteries to ensure the supply of oxygen and nutrients to the growing placenta and fetus. EVTs are peculiar in that they express no HLA-A and -B, but do express HLA-C, -E, and -G. Interestingly, KIR2DL1/S1 and KIR2DL2/3/S2 expression is more frequent on uNK cells than on matched peripheral blood NK cells (93), suggesting a tissue-specific role for KIRs on the uNK cells (which also express KIR2DL4, NKG2A, and LILRB1). The invading EVT reside in the same anatomical location as uNK cells and it has been hypothesized that KIR/KIR ligand combinations thus affect implantation and pregnancy success. In support of this, mothers lacking activating KIR genes (haplotype A/A), who consequently express only inhibitory KIR2DL1, in combination with a HLA-C2 ${ }^{+}$fetus, were at higher risk for preeclampsia and IUGR (94). In contrast, presence of the activating KIR2DS1 gene was protective, which indicated that activation of uNK cells via aKIR might be important in the interaction with EVT. Indeed, the same group recently showed that KIR2DS1 is functional on uNK cells (95). KIR2DS1 had an educating effect on uNK cells in HLA-C2 donors with regard to target cell induced degranulation, similar to the effect described for peripheral blood NK cells (15). However, signaling via KIR2DS1 on uNK cells also led to production of GM-CSF that could be used to attract trophoblasts in vitro. Together this provides mechanistic insight into the in vivo importance for activating KIR on uNK cells, in their potential interaction with EVT. Regarding other aKIRs on uNK cells, KIR2DS4 is also expressed on uNK cells, and using clonally expanded uNK cells, KIR2DS4 has been shown also to be functional (96). Future experiments will reveal whether KIR2DS4 plays a similar role as KIR2DS1 in the regulation of uNK cell function. HLA-G is expressed by trophoblasts and KIR2DL4 is also expressed by uNK cells. Expression of soluble HLA-G during pregnancy has been documented (92) and correlates with successful pregnancy (97). Consequently, intracellular KIR2DL4, engagement with its ligand HLA-G in endocytosis vesicles would result in production of various cytokines, which may have vascular remodeling consequences similar to KIR2DS1 activation (98).

\section{ACTIVATING KIR IN AUTOIMMUNE OF INFLAMMATORY DISEASE}

The relationships between KIR expression and autoimmune or inflammatory disorders remain largely unknown. Similarly to other diseases, the role of interaction between aKIRs and HLA class I has only been extrapolated from genetic studies looking both at $K I R$ and HLA gene expression. KIR2DS1 gene was suggested to be less common in patients with atopic dermatitis compared to healthy controls (99), suggesting that that KIR2DS1 might have a protective effect. In contrast, KIR2DS1 was found more often in patients with systemic lupus erythematous (100), and the same was true for KIR3DS1 in multiple sclerosis (MS) (101). Psoriasis vulgaris is also characterized by a clear association between KIR2DS1 and HLA-Cw6, whose co-carriage was over-represented in patients $(102,103)$. In this setting, Łuszczek et al. hypothesized that interactions between KIR2DS1 and its ligand would contribute to pathogenesis. Given that KIR2DS1 ${ }^{+}$NK cells are normally hyporesponsive in HLA-C2 positive donors, these studies indicate that there is a potential break of tolerance leading to psoriatic lesions. In contrast, a former study on psoriatic arthritis showed that KIR2DS1 and KIR2DS2 were associated with susceptibility to disease, but only in the absence of their cognate HLA ligands (104). Although responsiveness of KIR2DS1 ${ }^{+}$NK cells must be assessed, it is possible that an aKIRs education-dependent higher responsiveness would render KIR2DS1 ${ }^{+} \mathrm{NK}$ cells able to mediate autoimmune reactions, in the absence of HLA-C2, thus recognizing an unknown ligand.

\section{CONCLUDING REMARKS}

Although significant progress has been made in the understanding of aKIRs and their interactions with HLA class I, the role of aKIRs in disease and health still remains largely unexplored. The studies of aKIR/HLA class I associations indicate that aKIRs are likely to play a role in a number of different diseases, including infectious diseases, cancer, and autoimmunity. However, the lack of specific monoclonal antibodies to analyze expression patterns of aKIRs, together with their polymorphic nature and linkage 
disequilibrium with iKIRs have hampered detailed studies of aKIRs both in disease and in health. The development of specific antibodies, as well as the identification of ligands for aKIRs, potentially including non-HLA class I molecules, would greatly advance our understanding of aKIR biology. Finally, it is important to keep in mind that KIR expression is not restricted to NK cells but expression has also been documented on $\alpha \beta$ and $\gamma \delta$ T cells $(105,106)$. Particularly for $\alpha \beta$ T cells and infection or autoimmune diseases where polymorphism of HLA is important, the expression of aKIR might shed light on these genetic associations.

\section{REFERENCES}

1. Kiessling R, Klein E, Wigzell H. "Natural" killer cells in the mouse. I. Cytotoxic cells with specificity for mouse Moloney leukemia cells. Specificity and distribution according to genotype. Eur J Immunol (1975) 5:112-7. doi:10.1002/eji.1830050208

2. Herberman RB, Nunn ME, Holden HT, Lavrin DH. Natural cytotoxic reactivity of mouse lymphoid cells against syngeneic and allogeneic tumors. II. Characterization of effector cells. Int J Cancer (1975) 16:230-9. doi:10.1002/ ijc. 2910160205

3. Trinchieri G, Santoli D, Dee RR, Knowles BB. Anti-viral activity induced by culturing lymphocytes with tumor-derived or virus-transformed cells. Identification of the anti-viral activity as interferon and characterization of the human effector lymphocyte subpopulation. J Exp Med (1978) 147:1299-313. doi:10.1084/jem.147.5.1299

4. Becker S, Kiessling R, Lee N, Klein G. Modulation of sensitivity to natural killer cell lysis after in vitro explantation of a mouse lymphoma. J Natl Cancer Inst (1978) 61:1495-8.

5. Piontek GE, Taniguchi K, Ljunggren HG, Grönberg A, Kiessling R, Klein G, et al. YAC-1 MHC class I variants reveal an association between decreased NK sensitivity and increased $\mathrm{H}-2$ expression after interferon treatment or in vivo passage. J Immunol (1985) 135:4281-8.

6. Karre K, Ljunggren HG, Piontek G, Kiessling R. Selective rejection of H-2deficient lymphoma variants suggests alternative immune defence strategy. Nature (1986) 319:675-8. doi:10.1038/319675a0

7. Karlhofer FM, Ribaudo RK, Yokoyama WM. MHC class I alloantigen specificity of Ly-49+ IL-2-activated natural killer cells. Nature (1992) 358:66-70. doi:10.1038/358066a0

8. Moretta A, Vitale M, Bottino C, Orengo AM, Morelli L, Augugliaro R, et al. P58 molecules as putative receptors for major histocompatibility complex (MHC) class I molecules in human natural killer (NK) cells. Anti-p58 antibodies reconstitute lysis of MHC class I-protected cells in NK clones displaying different specificities. J Exp Med (1993) 178:597-604. doi:10.1084/jem.178.2.597

9. Moretta A, Tambussi G, Bottino C, Tripodi G, Merli A, Ciccone E, et al. A novel surface antigen expressed by a subset of human CD3- CD16+ natural killer cells. Role in cell activation and regulation of cytolytic function. J Exp Med (1990) 171:695-714. doi:10.1084/jem.171.3.695

10. Moretta A, Bottino C, Pende D, Tripodi G, Tambussi G, Viale O, et al. Identification of four subsets of human CD3-CD16+ natural killer (NK) cells by the expression of clonally distributed functional surface molecules: correlation between subset assignment of NK clones and ability to mediate specific alloantigen recognition. J Exp Med (1990) 172:1589-98. doi:10.1084/jem.172.6.1589

11. Litwin V, Gumperz J, Parham P, Phillips JH, Lanier LL. NKB1: a natural killer cell receptor involved in the recognition of polymorphic HLA-B molecules. J Exp Med (1994) 180:537-43. doi:10.1084/jem.180.2.537

12. Moretta A, Sivori S, Vitale M, Pende D, Morelli L, Augugliaro R, et al. Existence of both inhibitory ( $\mathrm{p} 58$ ) and activatory ( $\mathrm{p} 50$ ) receptors for HLAC molecules in human natural killer cells. J Exp Med (1995) 182:875-84. doi: $10.1084 /$ jem.182.3.875

13. Colonna M, Samaridis J. Cloning of immunoglobulin-superfamily members associated with HLA-C and HLA-B recognition by human natural killer cells. Science (1995) 268:405-8. doi:10.1126/science.7716543

14. Morvan M, David G, Sébille V, Perrin A, Gagne K, Willem C, et al. Autologous and allogeneic HLA KIR ligand environments and activating KIR control KIR NK-cell functions. Eur J Immunol (2008) 38:3474-86. doi:10.1002/eji. 200838407
15. Fauriat C, Ivarsson MA, Ljunggren H-G, Malmberg K-J, Michaëlsson J. Education of human natural killer cells by activating killer cell immunoglobulin-like receptors. Blood (2010) 115:1166-74. doi:10.1182/blood-2009-09-245746

16. David G, Morvan M, Gagne K, Kerdudou N, Willem C, Devys A, et al. Discrimination between the main activating and inhibitory killer cell immunoglobulinlike receptor positive natural killer cell subsets using newly characterized monoclonal antibodies. Immunology (2009) 128:172-84. doi:10.1111/j.1365-2567. 2009.03085.x

17. Béziat V, Liu LL, Malmberg J-A, Ivarsson MA, Sohlberg E, Björklund AT, et al. NK cell responses to cytomegalovirus infection lead to stable imprints in the human KIR repertoire and involve activating KIRs. Blood (2013) 121:2678-88. doi:10.1182/blood-2012-10-459545

18. Liu J, Xiao Z, Ko HL, Shen M, Ren EC. Activating killer cell immunoglobulinlike receptor 2DS2 binds to HLA-A*11. Proc Natl Acad Sci U S A (2014) 111:2662-7. doi:10.1073/pnas.1322052111

19. VandenBussche CJ, Mulrooney TJ, Frazier WR, Dakshanamurthy S, Hurley CK. Dramatically reduced surface expression of NK cell receptor KIR2DS3 is attributed to multiple residues throughout the molecule. Genes Immun (2009) 10:162-73. doi:10.1038/gene.2008.91

20. Graef T, Moesta AK, Norman PJ, Abi-Rached L, Vago L, Older Aguilar AM, et al. KIR2DS4 is a product of gene conversion with KIR3DL2 that introduced specificity for HLA-A 11 while diminishing avidity for HLA-C. J Exp Med (2009) 206:2557-72. doi:10.1084/jem.20091010

21. Steiner NK, Dakshanamurthy S, VandenBussche CJ, Hurley CK. Extracellular domain alterations impact surface expression of stimulatory natural killer cell receptor KIR2DS5. Immunogenetics (2008) 60:655-67. doi:10.1007/s00251008-0322-2

22. Della Chiesa M, Romeo E, Falco M, Balsamo M, Augugliaro R, Moretta L, et al. Evidence that the KIR2DS5 gene codes for a surface receptor triggering natural killer cell function. Eur J Immunol (2008) 38:2284-9. doi:10.1002/eji. 200838434

23. Czaja K, Borer A-S, Schmied L, Terszowski G, Stern M, Gonzalez A. A comprehensive analysis of the binding of anti-KIR antibodies to activating KIRs. Genes Immun (2013) 15(1):33-7. doi:10.1038/gene.2013.58

24. Carr WH, Rosen DB, Arase H, Nixon DF, Michaëlsson J, Lanier LL. Cutting Edge: KIR3DS1, a gene implicated in resistance to progression to AIDS, encodes a DAP12-associated receptor expressed on NK cells that triggers NK cell activation. J Immunol (2007) 178:647-51.

25. Moesta AK, Norman PJ, Yawata M, Yawata N, Gleimer M, Parham P. Synergistic polymorphism at two positions distal to the ligand-binding site makes KIR2DL2 a stronger receptor for HLA-C than KIR2DL3. J Immunol (2008) 180:3969-79.

26. Apps R, Gardner L, Moffett A. A critical look at HLA-G. Trends Immunol (2008) 29:313-21. doi:10.1016/j.it.2008.02.012

27. Cisneros E, Moraru M, Gómez-Lozano N, López-Botet M, Vilches C. KIR2DL5: an orphan inhibitory receptor displaying complex patterns of polymorphism and expression. Front Immunol (2012) 3:289. doi:10.3389/fimmu.2012.00289

28. Stern M, Ruggeri L, Capanni M, Mancusi A, Velardi A. Human leukocyte antigens A23, A24, and A32 but not A25 are ligands for KIR3DL1. Blood (2008) 112:708-10. doi:10.1182/blood-2008-02-137521

29. Dohring C, Scheidegger D, Samaridis J, Cella M, Colonna M. A human killer inhibitory receptor specific for HLA-A1,2. J Immunol (1996) 156(9):3098-101.

30. Pende D, Biassoni R, Cantoni C, Verdiani S, Falco M, di Donato C, et al. The natural killer cell receptor specific for HLA-A allotypes: a novel member of the p58/p70 family of inhibitory receptors that is characterized by three immunoglobulin-like domains and is expressed as a $140-\mathrm{kD}$ disulphide-linked dimer. J Exp Med (1996) 184:505-18. doi:10.1084/jem.184.2.505

31. Trundley AE, Hiby SE, Chang C, Sharkey AM, Santourlidis S, Uhrberg M, et al. Molecular characterization of KIR3DL3. Immunogenetics (2006) 57:904-16. doi:10.1007/s00251-005-0060-7

32. Parham P, Norman PJ, Abi-Rached L, Guethlein LA. Variable NK cell receptors exemplified by human KIR3DL1/S1. J Immunol (2011) 187:11-9. doi:10.4049/ jimmunol.0902332

33. Takeshita LYC, Gonzalez-Galarza FF, dos Santos EJM, Maia MHT, Rahman MM, Zain SMS, et al. A database for curating the associations between killer cell immunoglobulin-like receptors and diseases in worldwide populations. Database (Oxford) (2013) 2013:bat021. doi:10.1093/database/bat021

34. Pascal V, Yamada E, Martin MP, Alter G, Altfeld M, Metcalf JA, et al. Detection of KIR3DS1 on the cell surface of peripheral blood NK cells facilitates 
identification of a novel null allele and assessment of KIR3DS1 expression during HIV-1 infection. J Immunol (2007) 179:1625-33.

35. Trundley A, Frebel H, Jones D, Chang C, Trowsdale J. Allelic expression patterns of KIR3DS1 and 3DL1 using the Z27 and DX9 antibodies. Eur J Immunol (2007) 37:780-7. doi:10.1002/eji.200636773

36. Morvan M, Willem C, Gagne K, Kerdudou N, David G, Sebille V, et al. Phenotypic and functional analyses of KIR3DL1+ and KIR3DS1+ NK cell subsets demonstrate differential regulation by Bw4 molecules and induced KIR3DS1 expression on stimulated NK cells. J Immunol (2009) 182:6727-35. doi:10.4049/jimmunol.0900212

37. Cognet C, Farnarier C, Gauthier L, Frassati C, André P, Magérus-Chatinet A, et al. Expression of the HLA-C2-specific activating killer-cell Ig-like receptor KIR2DS1 on NK and T cells. Clin Immunol (2010) 135:26-32. doi:10.1016/j. clim.2009.12.009

38. O'Connor GM, Guinan KJ, Cunningham RT, Middleton D, Parham P, Gardiner CM. Functional polymorphism of the KIR3DL1/S1 receptor on human NK cells. J Immunol (2007) 178:235-41.

39. Rajagopalan S, Long EO. KIR2DL4 (CD158d): an activation receptor for HLAG. Front Immunol (2012) 3:258. doi:10.3389/fimmu.2012.00258

40. Goodridge JP, Witt CS, Christiansen FT, Warren HS. KIR2DL4 (CD158d) genotype influences expression and function in NK cells. J Immunol (2003) 171:1768-74.

41. Kikuchi-Maki A, Yusa S-I, Catina TL, Campbell KS. KIR2DL4 is an IL-2regulated NK cell receptor that exhibits limited expression in humans but triggers strong IFN-gamma production. J Immunol (2003) 171:3415-25.

42. David G, Djaoud Z, Willem C, Legrand N, Rettman P, Gagne K, et al. Large spectrum of HLA-C recognition by killer Ig-like receptor (KIR)2DL2 and KIR2DL3 and restricted C1 specificity of KIR2DS2: dominant impact of KIR2DL2/KIR2DS2 on KIR2D NK cell repertoire formation. J Immunol (2013) 191:4778-88. doi:10.4049/jimmunol.1301580

43. Stewart CA, Laugier-Anfossi F, Vély F, Saulquin X, Riedmuller J, Tisserant A, et al. Recognition of peptide-MHC class I complexes by activating killer immunoglobulin-like receptors. Proc Natl Acad Sci U S A (2005) 102:13224-9. doi:10.1073/pnas.0503594102

44. Moesta AK, Parham P. Diverse functionality among human NK cell receptors for the C1 epitope of HLA-C: KIR2DS2, KIR2DL2, and KIR2DL3. Front Immunol (2012) 3:336. doi:10.3389/fimmu.2012.00336

45. Rajagopalan S, Long EO. The direct binding of a p58 killer cell inhibitory receptor to human histocompatibility leukocyte antigen (HLA)-Cw4 exhibits peptide selectivity. J Exp Med (1997) 185:1523-8. doi:10.1084/jem.185.8.1523

46. Katz G, Gazit R, Arnon TI, Gonen-Gross T, Tarcic G, Markel G, et al. MHC class I-independent recognition of NK-activating receptor KIR2DS4. J Immunol (2004) 173:1819-25.

47. Smith HRC, Heusel JW, Mehta IK, Kim S, Dorner BG, Naidenko OV, et al. Recognition of a virus-encoded ligand by a natural killer cell activation receptor. Proc Natl Acad Sci U S A (2002) 99:8826-31. doi:10.1073/pnas.092258599

48. Arase H, Mocarski ES, Campbell AE, Hill AB, Lanier LL. Direct recognition of cytomegalovirus by activating and inhibitory NK cell receptors. Science (2002) 296:1323-6. doi:10.1126/science.1070884

49. Kielczewska A, Pyzik M, Sun T, Krmpotic A, Lodoen M, Munks M, et al. Ly49P recognition of cytomegalovirus-infected cells expressing H2-Dk and CMVencoded m04 correlates with the NK cell antiviral response. J Exp Med (2009) 206(3):515-23. doi:10.1084/jem.20080954

50. Fadda L, Borhis G, Ahmed P, Cheent K, Pageon SV, Cazaly A, et al. Peptide antagonism as a mechanism for NK cell activation. Proc Natl Acad Sci U S A (2010) 107:10160-5. doi:10.1073/pnas.0913745107

51. Hansasuta P, Dong T, Thananchai H, Weekes M, Willberg C, Aldemir H, et al. Recognition of HLA-A3 and HLA-A11 by KIR3DL2 is peptide-specific. Eur J Immunol (2004) 34(6):1673-9. doi:10.1002/eji.200425089

52. Lu X, Gibbs JS, Hickman HD, David A, Dolan BP, Jin Y, et al. Endogenous viral antigen processing generates peptide-specific MHC class I cell-surface clusters. Proc Natl Acad Sci U S A (2012) 109:15407-12. doi:10.1073/pnas.1208696109

53. Lanier LL, Corliss BC, Wu J, Leong C, Phillips JH. Immunoreceptor DAP12 bearing a tyrosine-based activation motif is involved in activating NK cells. Nature (1998) 391:703-7. doi:10.1038/35642

54. Olcese L, Cambiaggi A, Semenzato G, Bottino C, Moretta A, Vivier E. Human killer cell activatory receptors for MHC class I molecules are included in a multimeric complex expressed by natural killer cells. J Immunol (1997) 158:5083-6.
55. Turnbull IR, Colonna M. Activating and inhibitory functions of DAP12. Nat Rev Immunol (2007) 7:155-61. doi:10.1038/nri2014

56. Alter G, Martin MP, Teigen N, Carr WH, Suscovich TJ, Schneidewind A, et al. Differential natural killer cell-mediated inhibition of HIV-1 replication based on distinct KIR/HLA subtypes. J Exp Med (2007) 204:3027-36. doi:10.1084/jem.20070695

57. Biassoni R, Pessino A, Malaspina A, Cantoni C, Bottino C, Sivori S, et al. Role of amino acid position 70 in the binding affinity of p50.1 and p58.1 receptors for HLA-Cw4 molecules. Eur J Immunol (1997) 27:3095-9. doi:10.1002/eji. 1830271203

58. Chewning JH, Gudme CN, Hsu KC, Selvakumar A, Dupont B. KIR2DS1positive NK cells mediate alloresponse against the C2 HLA-KIR ligand group in vitro. J Immunol (2007) 179:854-68.

59. Pittari G, Liu X-R, Selvakumar A, Zhao Z, Merino E, Huse M, et al. NK cell tolerance of self-specific activating receptor KIR2DS1 in individuals with cognate HLA-C2 ligand. J Immunol (2013) 190(9):4650-60. doi:10.4049/jimmunol. 1202120

60. Kim S, Poursine-Laurent J, Truscott SM, Lybarger L, Song Y-J, Yang L, et al. Licensing of natural killer cells by host major histocompatibility complex class I molecules. Nature (2005) 436:709-13. doi:10.1038/nature03847

61. Anfossi N, André P, Guia S, Falk CS, Roetynck S, Stewart CA, et al. Human NK cell education by inhibitory receptors for MHC class I. Immunity (2006) 25:331-42. doi:10.1016/j.immuni.2006.06.013

62. Coudert JD, Zimmer J, Tomasello E, Cebecauer M, Colonna M, Vivier E, et al. Altered NKG2D function in NK cells induced by chronic exposure to NKG2D ligand-expressing tumor cells. Blood (2005) 106:1711-7. doi:10.1182/blood2005-03-0918

63. Sun JC, Lanier LL. Tolerance of NK cells encountering their viral ligand during development. J Exp Med (2008) 205:1819-28. doi:10.1084/jem.20072448

64. Narni-Mancinelli E, Jaeger BN, Bernat C, Fenis A, Kung S, De Gassart A, et al. Tuning of natural killer cell reactivity by NKp46 and Helios calibrates T cell responses. Science (2012) 335:344-8. doi:10.1126/science.1215621

65. Kumar V, McNerney ME. A new self: MHC-class-I-independent natural-killercell self-tolerance. Nat Rev Immunol (2005) 5:363-74. doi:10.1007/s11626013-9593-5

66. Bolanos FD, Tripathy SK. Activation receptor-induced tolerance of mature NK cells in vivo requires signaling through the receptor and is reversible. JImmunol (2011) 186:2765-71. doi:10.4049/jimmunol.1003046

67. Joncker NT, Shifrin N, Delebecque F, Raulet DH. Mature natural killer cells reset their responsiveness when exposed to an altered MHC environment. J Exp Med (2010) 207:2065-72. doi:10.1084/jem.20100570

68. Pradeu T, Jaeger S, Vivier E. The speed of change: towards a discontinuity theory of immunity? Nat Rev Immunol (2013) 13:764-9. doi:10.1038/nri3521

69. Körner C, Altfeld M. Role of KIR3DS1 in human diseases. Front Immunol (2012) 3:326. doi:10.3389/fimmu.2012.00326

70. Martin MP, Gao X, Lee J-H, Nelson GW, Detels R, Goedert JJ, et al. Epistatic interaction between KIR3DS1 and HLA-B delays the progression to AIDS. Nat Genet (2002) 31:429-34.

71. Pelak K, Need AC, Fellay J, Shianna KV, Feng S, Urban TJ, et al. Copy number variation of KIR genes influences HIV-1 control. PLoS Biol (2011) 9:e1001208. doi:10.1371/journal.pbio. 1001208

72. Boulet S, Sharafi S, Simic N, Bruneau J, Routy J-P, Tsoukas CM, et al. Increased proportion of KIR3DS1 homozygotes in HIV-exposed uninfected individuals. AIDS (2008) 22:595-9. doi:10.1097/QAD.0b013e3282f56b23

73. Alter G, Rihn S, Walters K, Nolting A, Martin M, Rosenberg E, et al. HLA class I subtype dependent expansion of KIR3DS1+ and KIR3DL1+ NK cells during acute HIV-1 infection. J Virol (2009) 83(13):6798-805. doi:10.1128/ JVI.00256-09

74. Gaudieri S, DeSantis D, McKinnon E, Moore C, Nolan D, Witt CS, et al. Killer immunoglobulin-like receptors and HLA act both independently and synergistically to modify HIV disease progression. Genes Immun (2005) 6:683-90. doi:10.1038/sj.gene.6364256

75. Long BR, Ndhlovu LC, Oksenberg JR, Lanier LL, Hecht FM, Nixon DF, et al. Conferral of enhanced natural killer cell function by KIR3DS1 in early human immunodeficiency virus type 1 infection. J Virol (2008) 82:4785-92. doi:10.1128/JVI.02449-07

76. Dring MM, Morrison MH, McSharry BP, Guinan KJ, Hagan R, Consortium IHR, et al. Innate immune genes synergize to predict increased risk of 
chronic disease in hepatitis C virus infection. Proc Natl Acad Sci U S A (2011) 108:5736-41. doi:10.1073/pnas.1016358108

77. de Vasconcelos JM, de Jesus Maués Pereira Móia L, Amaral Ido S, Miranda EC, Cicalisetakeshita LY, de Oliveira LF, et al. Association of killer cell immunoglobulin-like receptor polymorphisms with chronic hepatitis $\mathrm{C}$ and responses to therapy in Brazil. Genet Mol Biol (2013) 36:22-7. doi:10.1590/ S1415-47572013000100004

78. Jennes W, Verheyden S, Demanet C, Menten J, Vuylsteke B, Nkengasong JN, et al. Low CD4+ T cell counts among African HIV-1 infected subjects with group B KIR haplotypes in the absence of specific inhibitory KIR ligands. PLoS One (2011) 6:e17043. doi:10.1371/journal.pone.0017043

79. Cohen GB, Gandhi RT, Davis DM, Mandelboim O, Chen BK, Strominger JL, et al. The selective downregulation of class I major histocompatibility complex proteins by HIV-1 protects HIV-infected cells from NK cells. Immunity (1999) 10:661-71. doi:10.1016/S1074-7613(00)80065-5

80. Wauquier N, Padilla C, Becquart P, Leroy E, Vieillard V. Association of KIR2DS1 and KIR2DS3 with fatal outcome in Ebola virus infection. Immunogenetics (2010) 62:767-71. doi:10.1007/s00251-010-0480-x

81. Marcenaro E, Pesce S, Sivori S, Carlomagno S, Moretta L, Moretta A. KIR2DS1dependent acquisition of CCR7 and migratory properties by human NK cells interacting with allogeneic HLA-C2+ DCs or T-cell blasts. Blood (2013) 121:3396-401. doi:10.1182/blood-2012-09-458752

82. Ozturk OG, Gun FD, Polat G. Killer cell immunoglobulin-like receptor genes in patients with breast cancer. Med Oncol (2012) 29:511-5. doi:10.1007/s12032011-9932-x

83. Marin D, Gabriel IH, Ahmad S, Foroni L, de Lavallade H, Clark R, et al. KIR2DS1 genotype predicts for complete cytogenetic response and survival in newly diagnosed chronic myeloid leukemia patients treated with imatinib. Leukemia (2012) 26:296-302. doi:10.1038/leu.2011.180

84. Gabriel IH, Sergeant R, Szydlo R, Apperley JF, deLavallade H, Alsuliman A, et al. Interaction between KIR3DS1 and HLA-Bw4 predicts for progressionfree survival after autologous stem cell transplantation in patients with multiple myeloma. Blood (2010) 116:2033-9. doi:10.1182/blood-2010-03273706

85. Karabon L, Jedynak A, Giebel S, Wołowiec D, Kielbinski M, Woszczyk D, et al. KIR/HLA gene combinations influence susceptibility to B-cell chronic lymphocytic leukemia and the clinical course of disease. Tissue Antigens (2011) 78:129-38. doi:10.1111/j.1399-0039.2011.01721.x

86. Venstrom JM, Pittari G, Gooley TA, Chewning JH, Spellman S, Haagenson $\mathrm{M}$, et al. HLA-C-dependent prevention of leukemia relapse by donor activating KIR2DS1. N Engl J Med (2012) 367:805-16. doi:10.1056/ NEJMoal200503

87. Cooley S, Trachtenberg E, Bergemann TL, Saeteurn K, Klein J, Le CT, et al. Donors with group B KIR haplotypes improve relapse-free survival after unrelated hematopoietic cell transplantation for acute myelogenous leukemia. Blood (2009) 113:726-32. doi:10.1182/blood-2008-07-171926

88. Cooley S, Weisdorf DJ, Guethlein LA, Klein JP, Wang T, Le CT, et al. Donor selection for natural killer cell receptor genes leads to superior survival after unrelated transplantation for acute myelogenous leukemia. Blood (2010) 116:2411-9. doi:10.1182/blood-2010-05-283051

89. McQueen KL, Dorighi KM, Guethlein LA, Wong R, Sanjanwala B, Parham P. Donor-recipient combinations of group A and B KIR haplotypes and HLA class I ligand affect the outcome of HLA-matched, sibling donor hematopoietic cell transplantation. Hum Immunol (2007) 68:309-23. doi:10.1016/j.humimm. 2007.01.019

90. Pende D, Marcenaro S, Falco M, Martini S, Bernardo ME, Montagna D, et al. Anti-leukemia activity of alloreactive NK cells in KIR ligand-mismatched haploidentical HSCT for pediatric patients: evaluation of the functional role of activating KIR and redefinition of inhibitory KIR specificity. Blood (2009) 113:3119-29. doi:10.1182/blood-2008-06-164103

91. Moffett-King A. Natural killer cells and pregnancy. Nat Rev Immunol (2002) 2:656-63. doi:10.1038/nri886

92. Moffett A, Loke C. Immunology of placentation in eutherian mammals. Nat Rev Immunol (2006) 6:584-94. doi:10.1038/nri1897
93. Male V, Sharkey A, Masters L, Kennedy PR, Farrell LE, Moffett A. The effect of pregnancy on the uterine NK cell KIR repertoire. Eur J Immunol (2011) 41:3017-27. doi:10.1002/eji.201141445

94. Hiby SE, Apps R, Sharkey AM, Farrell LE, Gardner L, Mulder A, et al. Maternal activating KIRs protect against human reproductive failure mediated by fetal HLA-C2. J Clin Invest (2010) 120:4102-10. doi:10.1172/JCI43998

95. Xiong S, Sharkey AM, Kennedy PR, Gardner L, Farrell LE, Chazara O, et al. Maternal uterine NK cell-activating receptor KIR2DS1 enhances placentation. J Clin Invest (2013) 123:4264-72. doi:10.1172/JCI68991

96. Hanna J, Goldman-Wohl D, Hamani Y, Avraham I, Greenfield C, NatansonYaron S, et al. Decidual NK cells regulate key developmental processes at the human fetal-maternal interface. Nat Med (2006) 12:1065-74. doi:10.1038/ $\mathrm{nm} 1452$

97. Rizzo R, Vercammen M, van de Velde H, Horn PA, Rebmann V. The importance of HLA-G expression in embryos, trophoblast cells, and embryonic stem cells. Cell Mol Life Sci (2011) 68:341-52. doi:10.1007/s00018-010-0578-1

98. Rajagopalan S, Bryceson YT, Kuppusamy SP, Geraghty DE, van der Meer A, Joosten I, et al. Activation of NK cells by an endocytosed receptor for soluble HLA-G. PLoS Biol (2006) 4:e9. doi:10.1371/journal.pbio.0040009

99. Niepiekło-Miniewska W, Majorczyk E, Matusiak L, Gendzekhadze K, Nowak I, Narbutt J, et al. Protective effect of the KIR2DS1 gene in atopic dermatitis. Gene (2013) 527:594-600. doi:10.1016/j.gene.2013.06.015

100. Hou Y-F, Zhang Y-C, Jiao Y-L, Wang L-C, Li J-F, Pan Z-L, et al. Disparate distribution of activating and inhibitory killer cell immunoglobulin-like receptor genes in patients with systemic lupus erythematosus. Lupus (2010) 19:20-6. doi:10.1177/0961203309345779

101. García-León JA, Pinto-Medel MJ, García-Trujillo L, López-Gómez C, Maldonado-Sanchez R, Fernández-Fernández Ó, et al. Killer cell immunoglobulin-like receptor genes in Spanish multiple sclerosis patients. Mol Immunol (2011) 48:1896-902. doi:10.1016/j.molimm.2011.05.018

102. Guðjónsson JE, Karason A, Antonsdottir AA, Rúnarsdottir EH, Gulcher JR, Stefansson K, et al. HLA-Cw6-positive and HLA-Cw6-negative patients with psoriasis vulgaris have distinct clinical features. J Invest Dermatol (2002) 118:362-5. doi:10.1046/j.0022-202x.2001.01656.x

103. Łuszczek W, Manczak M, Cisło M, Nockowski P, Wisniewski A, Jasek M, et al. Gene for the activating natural killer cell receptor, KIR2DS1, is associated with susceptibility to psoriasis vulgaris. Hum Immunol (2004) 65:758-66. doi:10.1016/j.humimm.2004.05.008

104. Martin MP, Nelson G, Lee J-H, Pellett F, Gao X, Wade J, et al. Cutting edge: susceptibility to psoriatic arthritis: influence of activating killer Ig-like receptor genes in the absence of specific HLA-C alleles. J Immunol (2002) 169:2818-22.

105. Bjorkstrom NK, Beziat V, Cichocki F, Liu LL, Levine J, Larsson S, et al. CD8 T cells express randomly selected KIRs with distinct specificities compared with NK cells. Blood (2012) 120:3455-65. doi:10.1182/blood-2012-03-416867

106. Halary F, Peyrat MA, Champagne E, López-Botet M, Moretta A, Moretta L, et al. Control of self-reactive cytotoxic $\mathrm{T}$ lymphocytes expressing gamma delta T cell receptors by natural killer inhibitory receptors. Eur J Immunol (1997) 27:2812-21. doi:10.1002/eji.1830271111

Conflict of Interest Statement: The authors declare that the research was conducted in the absence of any commercial or financial relationships that could be construed as a potential conflict of interest.

Received: 23 January 2014; accepted: 07 April 2014; published online: 22 April 2014. Citation: Ivarsson MA, Michaëlsson J and Fauriat C (2014) Activating killer cell Ig-like receptors in health and disease. Front. Immunol. 5:184. doi: 10.3389/fimmu.2014.00184 This article was submitted to NK Cell Biology, a section of the journal Frontiers in Immunology.

Copyright $\odot 2014$ Ivarsson, Michaëlsson and Fauriat. This is an open-access article distributed under the terms of the Creative Commons Attribution License (CC BY). The use, distribution or reproduction in other forums is permitted, provided the original author(s) or licensor are credited and that the original publication in this journal is cited, in accordance with accepted academic practice. No use, distribution or reproduction is permitted which does not comply with these terms. 